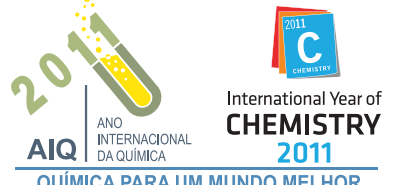

\title{
PRÉ-CONCENTRAÇÃO DE Cr(III) E ANÁLISE DE ESPECIAÇÃO DE CROMO EMPREGANDO MONTMORILONITA SATURADA COM ÍONS POTÁSSIO
}

\author{
Sérgio Ribeiro Frois, Marco Tadeu Grassi, Tatyane Caruso Fernandes, Ronaldo Adriano dos Santos Barreto e Gilberto Abate* \\ Departamento de Química, Universidade Federal do Paraná, CP 19081, 81531-990 Curitiba - PR, Brasil
}

Recebido em 8/7/10; aceito em 26/10/10; publicado na web em 26/1/11

\begin{abstract}
PRECONCENTRATION OF Cr(III) AND SPECIATION ANALYSIS OF CHROMIUM EMPLOYING MONTMORILLONITE SATURATED WITH POTASSIUM IONS. The clay mineral montmorillonite-K10 (MT), treated under acidic medium and saturated with potassium ions (MTK), was employed in sorption and desorption studies aiming the preconcentration of $\mathrm{Cr}(\mathrm{III})$ and the speciation analysis of chromium. The sorption process of $\mathrm{Cr}$ (III) was close to $100 \%$, suggesting that MTK was a good material for $\mathrm{Cr}$ (III) preconcentration, although, the maximum recovery in $\mathrm{HNO}_{3}$ solution was near $89 \%$. On the other hand, $\mathrm{Cr}(\mathrm{VI})$ practically was not retained in MTK, suggesting this material as an appropriate mineral phase to be used in speciation analysis of chromium in aqueous medium.
\end{abstract}

Keywords: clay minerals; sorption; speciation analysis.

\section{INTRODUÇÃO}

Algumas espécies metálicas podem apresentar efeitos altamente tóxicos quando presentes em suas formas iônicas livres ou combinadas. Geralmente, elas surgem a partir de fontes geogênicas ou antropogênicas, sendo essa última responsável pela contaminação de diversos compartimentos ambientais, afetando em consequência animais, peixes, plantas e o próprio homem. ${ }^{1,2}$ Entre os metais largamente utilizados para fins industriais, especial destaque pode ser dado ao cromo, o qual está presente em efluentes aquosos ou sólidos nas formas iônicas como $\mathrm{Cr}(\mathrm{III})$ ou $\mathrm{Cr}(\mathrm{VI})$. Dentre as atividades industriais potenciais geradoras de efluentes contendo tais espécies, pode-se citar: eletrodeposição, curtimento do couro, preservação de madeira, tintas, têxteis e metalúrgicas. O cromo também pode estar presente em águas de torres de resfriamento e em fases lixiviadas de aterros sanitários. - $^{3-5}$

O Cr(III) está envolvido na manutenção dos níveis de glicose, colesterol e triglicerídeos, desempenhando, portanto, um papel essencial como nutriente aos organismos vivos. ${ }^{4,6-8}$ Ao contrário, a forma hexavalente apresenta elevada toxicidade, estando relacionada a casos clínicos como irritação e ulceração nasal, reações de hipersensibilidade e dermatite por contato, sendo a dose letal $\left(\mathrm{DL}_{50}\right)$ entre 50 e $100 \mathrm{mg} \mathrm{kg}^{-1}$, valor esse muito inferior quando comparado com a forma trivalente, cujos valores se situam entre 1900 e $3300 \mathrm{mg}$ $\mathrm{kg}^{-1}$, em ambos os casos considerando ingestão oral em ratos. ${ }^{9}$ Além disso, o $\mathrm{Cr}(\mathrm{VI})$ é uma espécie carcinogênica, em decorrência da fácil permeação na membrana celular e do elevado potencial oxidante. ${ }^{8,9}$

No Brasil os limites de lançamento de efluentes em corpos aquáticos são determinados conforme a Resolução 397/2008 do Conselho Nacional do Meio Ambiente, ${ }^{10}$ sendo que os efluentes somente poderão ser lançados nos corpos aquáticos receptores quando os teores de $\mathrm{Cr}(\mathrm{VI})$ e $\mathrm{Cr}$ (III) forem inferiores a 0,1 e $1,0 \mathrm{mg} \mathrm{L}^{-1}$, respectivamente.

Em virtude da grande utilização na indústria e da diferença de toxicidade de ambas as espécies, torna-se imprescindível o estabelecimento de métodos analíticos direcionados para a quantificação das duas formas ou principalmente do $\mathrm{Cr}(\mathrm{VI})$ em efluentes aquosos ou

\footnotetext{
*e-mail: gilbertoabate@ufpr.br
}

sólidos. Geralmente são empregadas técnicas analíticas como espectrometria de absorção molecular na região do visível e espectrometria de absorção ou de emissão atômica. Todavia, as duas últimas técnicas não permitem uma análise de especiação direta, sendo necessária a utilização de processos de separação de uma das duas espécies de cromo. Por outro lado, a técnica de absorção molecular na região do visível, usualmente, apresenta valores de limites de quantificação superiores. Dessa forma, a implementação de métodos envolvendo a separação e pré-concentração dessas espécies vem sendo largamente estudada, empregando as mais diversas abordagens, dentre as quais, pode-se citar: extração empregando ponto de nuvem $;{ }^{11}$ utilização de processos de coprecipitação; ${ }^{8,12}$ pré-concentração em resinas; $;^{7,13}$ sílica-gel modificada ${ }^{2,14}$ e, fase C-18. ${ }^{15}$

Fases minerais como óxidos e argilominerais apresentam propriedades como elevada área superficial $\left(700-800 \mathrm{~m}^{2} \mathrm{~g}^{-1}\right)$ e alta capacidade de troca catiônica $(80-200 \mathrm{mmol} / 100 \mathrm{~g}),{ }^{16-18}$ constituindo, portanto, atrativos materiais a serem investigados com propósitos analíticos. Todavia, os mesmos vêm sendo estudados com enfoque direcionado principalmente a tratamento de efluentes contendo íons de metais tóxicos. ${ }^{19-21}$ Em decorrência das propriedades já citadas desses materiais, que aliadas ao baixo custo, grande disponibilidade, boa inércia química e facilidade para purificação, é possível que essas fases minerais, após purificação, possam atuar como especiadores e pré-concentradores analíticos de metais tóxicos. Algumas propostas são relatadas na literatura, as quais, contudo, não utilizam argilominerais in natura, sendo previamente modificadas com algum tipo de ligante orgânico. .2-25 $^{-25}$

O objetivo do presente trabalho foi avaliar o potencial da fase mineral montmorilonita-K10 (Aldrich) no processo de sorção e de dessorção das espécies iônicas de cromo, visando a pré-concentração analítica do $\mathrm{Cr}$ (III) e a especiação entre as formas de $\mathrm{Cr}$ (III) e $\mathrm{Cr}(\mathrm{VI})$. Para tal, o material foi previamente purificado e tratado com íons potássio, sendo adotado para a quantificação do $\mathrm{Cr}(\mathrm{VI})$ o método empregando o reagente 1,5-difenilcarbazida, (DFC), o qual reage com a forma de $\mathrm{Cr}(\mathrm{VI})$. Cabe ressaltar que é de grande valia o desenvolvimento de métodos analíticos direcionados para diferenciação entre as espécies trivalente e hexavalente, especialmente em decorrência da grande diferença de toxicidade de ambas. Até o momento, não 
constam relatos na literatura propondo a utilização de argilominerais sem tratamento superficial, visando a diferenciação entre as duas espécies de cromo, para aplicações analíticas.

\section{PARTE EXPERIMENTAL}

\section{Equipamentos e materiais}

Para a determinação do comprimento de onda de máxima absorção do complexo cromo-DFC foi utilizado um espectrofotômetro da marca HP 8452A. Para as demais determinações espectrofotométricas foi empregado um equipamento da marca Femto, modelo 700 Plus, sendo utilizada uma cubeta em vidro com $1,0 \mathrm{~cm}$ de caminho ótico. As medidas de absorbância foram efetuadas em comprimento de onda de $540 \mathrm{~nm}$. Um difratômetro de Raios X Shimadzu XRD 6000, com radiação $\mathrm{K} \alpha$ do cobre $(\lambda=1,5418), 40 \mathrm{kV}-40 \mathrm{~mA}$, foi empregando visando caracterizar o material estudado. Para as medidas de $\mathrm{pH}$ foi utilizado um potenciômetro da marca Hanna, $\mathrm{pH}$ 21, dotado de um eletrodo de vidro combinado com eletrodo de referência Ag/ $\mathrm{AgCl}$, sendo o mesmo previamente calibrado com soluções tampão de $\mathrm{pH}$ 4,00 e 7,00. Foram empregados ainda outros equipamentos e acessórios auxiliares como centrífuga, agitador orbital, estufa, micropipetas, entre outros.

\section{Reagentes e soluções}

As soluções estoques individuais do $\mathrm{Cr}(\mathrm{VI})$ e $\mathrm{Cr}(\mathrm{III})$ foram preparadas dissolvendo-se quantidades apropriadas dos sais $\mathrm{K}_{2} \mathrm{Cr}_{2} \mathrm{O}_{7}$ (Riedel-de Haën) padrão primário e $\mathrm{CrCl}_{3} \cdot 6 \mathrm{H}_{2} \mathrm{O}$ (Merck), para o qual foi previamente determinado o percentual de pureza. As soluções foram preparadas em concentração de $500 \mathrm{mg} \mathrm{L}^{-1}$ para cada uma das espécies em questão. As soluções padrão foram obtidas a partir de diluições sucessivas da solução estoque. O reagente cromogênico 1,5-difenilcarbazida (DFC) foi preparado a partir da dissolução de 0,050 g de DFC (Rea-Tech) em $10 \mathrm{~mL}$ de acetona (Vetec) e armazenado a $4{ }^{\circ} \mathrm{C}$.

Toda a vidraria empregada foi previamente lavada com solução de $\mathrm{HNO}_{3}(5 \%$ v/v) e enxaguada com água destilada e deionizada, a qual foi obtida empregando o equipamento Simplicity da Millipore. Os demais reagentes empregados foram de grau analítico das marcas Merck, Aldrich, Carlo Erba, ou de qualidade similar.

Todas as fases sobrenadantes obtidas nos experimentos de sorção e de dessorção foram previamente filtradas, antes das determinações espectrofotométricas. Para tal, foram utilizadas membranas descartáveis de acetato de celulose da Millipore, com abertura de poro de 0,45 $\mu \mathrm{m}$ e $25 \mathrm{~mm}$ de diâmetro, com o auxílio de seringas hipodérmicas.

\section{Tratamento e caracterização da montmorilonita}

Foi utilizada a fase mineral montmorilonita K-10 (28152-2) fornecida pela Aldrich com área superficial 220-270 $\mathrm{m}^{2} \mathrm{~g}^{-1}$, identificada no presente trabalho como MT. Uma massa de $10 \mathrm{~g}$ de MT foi mantida em contato com $20 \mathrm{~mL}_{\text {de }} \mathrm{HNO}_{3} 2,0 \mathrm{~mol} \mathrm{~L}-1$, em um agitador orbital por $10 \mathrm{~min}$, sendo em seguida centrifugada por $10 \mathrm{~min}$ a 2800 rpm. Essa lavagem tem como função liberar eventuais íons metálicos existentes, bem como condicionar a MT, visto que o tratamento em meio ácido deve modificar a estrutura do material, e esse mesmo meio será empregado nos estudos posteriores de dessorção do Cr(III).

As fases sobrenadantes foram descartadas e a fase sólida de MT foi lavada com água deionizada por três vezes com agitação orbital por 10 min e posterior centrifugação, como anteriormente. As fases sobrenadantes foram descartadas e ao sólido foram adicionados 20 $\mathrm{mL}$ de solução de $\mathrm{KCl} 1,0 \mathrm{~mol} \mathrm{~L}^{-1}$, seguido de agitação por $30 \mathrm{~min}$ e centrifugação por $10 \mathrm{~min}$. Os sobrenadantes foram descartados e a MT foi tratada mais duas vezes com a solução de $\mathrm{KCl} 1,0 \mathrm{~mol} \mathrm{~L}^{-1}$. A fase sólida foi lavada com $40 \mathrm{~mL}$ de água deionizada por três vezes, seguido por 10 min de agitação e posterior centrifugação, como anteriormente. A fase sólida foi seca a $70{ }^{\circ} \mathrm{C}$ até massa constante, macerada em almofariz e novamente mantida para secagem. Parte da MT foi submetida à determinação da distribuição granulométrica, empregando-se peneiras da marca Bertel com as seguintes aberturas: $250,170,115,80$ e 60 mesh. Essa montmorilonita tratada com íons $\mathrm{K}^{+}$foi mantida em dessecador, sendo identificada como MTK. Esse material foi caracterizado por difração de raios X para determinação do espaçamento basal.

\section{Preparo das curvas analíticas para determinação de $\mathrm{Cr}(\mathrm{VI})$ e Cr(III) ${ }^{26}$}

As curvas analíticas para $\mathrm{Cr}(\mathrm{VI})$ foram obtidas a partir de uma solução estoque (500 $\mathrm{mg} \mathrm{L}^{-1} \mathrm{de} \mathrm{Cr}(\mathrm{VI})$ ). Foram preparados $10,00 \mathrm{~mL}$ das soluções nas seguintes concentrações $\left(\mathrm{mg} \mathrm{L}^{-1}\right)$ : 0,000 (prova em branco); 0,050; 0,100; 0,200; 0,400 e 0,800. Em cada um dos balões foram adicionadoa $200 \mu \mathrm{L}$ do complexante DFC em $\mathrm{H}_{2} \mathrm{SO}_{4} 0,10 \%$ (v/v). Os sinais analíticos foram obtidos por espectrofotometria em comprimento de onda de $540 \mathrm{~nm}$.

Para o Cr(III) foi realizada uma etapa prévia de oxidação. Em 5 béqueres de $50 \mathrm{~mL}$ foram adicionados volumes de solução de $\mathrm{Cr}$ (III) em concentração de $500 \mathrm{mg} \mathrm{L}^{-1}$, suficiente para perfazer as mesmas concentrações empregadas para o $\mathrm{Cr}(\mathrm{VI})$. Foram adicionados 100 $\mu \mathrm{L}$ de solução de $\mathrm{H}_{2} \mathrm{SO}_{4}\left(10 \%\right.$ v/v) e $50 \mu \mathrm{L}$ de $\mathrm{KMnO}_{4}\left(20 \mathrm{~g} \mathrm{~L}^{-1}\right)$, sendo a solução aquecida até a fervura. Após a permanência de uma tonalidade avermelhada na solução, ainda em aquecimento, foram adicionados $200 \mu \mathrm{L}$ de solução de azida sódica $\left(2,0 \mathrm{~g} \mathrm{~L}^{-1}\right)$, sendo a fervura mantida por mais $5 \mathrm{~min}$. Após resfriamento adicionou-se 50 $\mu \mathrm{L}$ de $\mathrm{H}_{3} \mathrm{PO}_{4}$ concentrado e $200 \mu \mathrm{L}$ do complexante DFC em $\mathrm{H}_{2} \mathrm{SO}_{4}$ $0,1 \%$ (v/v), sendo as determinações realizadas como anteriormente para o $\mathrm{Cr}(\mathrm{VI})$. Experimentos adicionais foram realizados em triplicata contendo 0,500 ou $0,050 \mathrm{mg} \mathrm{L}^{-1}$ de cada uma das espécies de cromo. As determinações foram realizadas sem utilizar a etapa de oxidação do $\mathrm{Cr}$ (III) e, em seguida, submetendo a mistura à etapa de oxidação, visando a determinação do teor total de cromo, utilizando os mesmos procedimentos já descritos.

\section{Estudo de variáveis relacionadas ao processo de sorção e dessorção em MTK}

Para determinar o melhor tempo de sorção do $\mathrm{Cr}$ (III) foram realizados experimentos em triplicata, utilizando 0,1000 $\mathrm{g}$ da MTK na presença de $10,00 \mathrm{~mL}$ de $\mathrm{Cr}$ (III) $0,500 \mathrm{mg} \mathrm{L}^{-1}$. As suspensões foram mantidas sob agitação orbital por 60, 10 e 0,5 min. Em todos os casos foi utilizado um tempo de 5 min de centrifugação (2800 rpm), sendo as amostras filtradas e o teor de $\mathrm{Cr}$ (III) quantificado pelo método da DFC após a etapa de oxidação. Cabe salientar que esse experimento, bem como os demais estudos de sorção e de dessorção descritos adiante, foram conduzidos no laboratório em temperatura ambiente, situada entre 18 e $25^{\circ} \mathrm{C}$.

Foi investigada a influência da massa de MTK no processo de sorção de $\mathrm{Cr}$ (III). Para tal, foi utilizada solução de $\mathrm{Cr}$ (III) em concentração inicial de $0,500 \mathrm{mg} \mathrm{L}^{-1} \mathrm{em}$ contato com 0,1000; 0,2000; 0,4000 e 0,5000 g de MTK, durante $10 \mathrm{~min}$ sob agitação orbital. $\mathrm{O}$ processo de sorção foi avaliado de forma similar àquela descrita anteriormente. Foram conduzidos experimentos em paralelo, empregando as mesmas massas de MTK, na ausência de íons Cr(III), a fim de verificar se haveria influência do aumento de massa de MTK nos sinais das provas em branco. 
Estudos de sorção e de dessorção com $10,00 \mathrm{~mL}$ de solução de $\mathrm{Cr}$ (III) em concentrações de 0,250; 0,500 e 1,000 $\mathrm{mg} \mathrm{L}^{-1}$ foram realizados, utilizando $0,1000 \mathrm{~g}$ de MTK, sendo as suspensões mantidas sob agitação orbital por $10 \mathrm{~min}$. As amostras foram centrifugadas, filtradas e as concentrações de $\mathrm{Cr}$ (III) determinadas como anteriormente. Em seguida, as fases sólidas foram mantidas em contato durante 10 min com 5,0 mL de solução de $\mathrm{HNO}_{3}$ em concentração 2,0 mol L-1, centrifugadas e filtradas, sendo o teor de $\mathrm{Cr}$ (III) quantificado como anteriormente, visando avaliar o processo de dessorção. Foi avaliado o tempo de dessorção na presença de solução de $\mathrm{HNO}_{3}$ em concentração 2,0 mol L-1 durante 10 e 60 min para a pré-concentração inicial de $\mathrm{Cr}(\mathrm{III})$ de $0,500 \mathrm{mg} \mathrm{L}^{-1}$. Foi investigada ainda a influência do efeito de ultrassom no processo de dessorção, sendo parte das amostras sonicadas por $15 \mathrm{~min}$, na potência de $120 \mathrm{~W}$.

Foi também avaliada a influência do $\mathrm{HNO}_{3}$ em concentração 2,0 mol L ${ }^{-1}$ sobre a MTK. Para tal, $0,1000 \mathrm{~g}$ da MTK foi submetida a sucessivos tratamentos com $5,0 \mathrm{~mL}$ do $\mathrm{HNO}_{3}$ sob agitação por 15 min, sendo que após cada ciclo de lavagem com o $\mathrm{HNO}_{3}$ a suspensão era centrifugada e um novo volume de $\mathrm{HNO}_{3}$ era adicionado para repetir o ciclo. Esse ciclo foi repetido por sete vezes, sendo realizados dois conjuntos de experimentos em diferentes dias. Todos os sobrenadantes (provas em branco) foram analisados empregando o método da DFC.

\section{Estudo de sorção e de dessorção do $\mathrm{Cr}$ (III) e $\mathrm{Cr}$ (VI) pela MTK}

Em três tubos de centrífuga de $15 \mathrm{~mL}$ (tubos A, B e C) foi transferida uma massa de $0,1000 \mathrm{~g}$ de MTK. No tubo A foram adicionados $10,00 \mathrm{~mL}$ de água deionizada; no tubo $\mathrm{B}, 10,00 \mathrm{~mL}$ de solução de $\mathrm{Cr}$ (III) em concentração de $0,500 \mathrm{mg} \mathrm{L}^{-1}$ e no tubo $\mathrm{C}, 10,00 \mathrm{~mL}$ de solução de $\mathrm{Cr}(\mathrm{VI})$ em concentração de $0,500 \mathrm{mg} \mathrm{L}^{-1}$. Os tubos foram mantidos em agitador orbital por 10 min e centrifugados por $10 \mathrm{~min}$ a $2800 \mathrm{rpm}$. As fases sobrenadantes foram filtradas, sendo as concentrações de $\mathrm{Cr}(\mathrm{III})$ ou de $\mathrm{Cr}(\mathrm{VI})$ determinadas como anteriormente. Às fases sólidas foi adicionado um volume de $5,0 \mathrm{~mL}$ de solução de

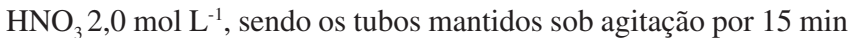
e centrifugados por $10 \mathrm{~min}$. Nas fases líquidas, previamente filtradas, foi determinada a concentração de $\mathrm{Cr}(\mathrm{III})$ ou de $\mathrm{Cr}$ (VI).

Em outro grupo de experimentos foi avaliado o processo de sorção com o $\mathrm{Cr}$ (III) e Cr(VI) juntos. Para tal, em quatro tubos de centrífuga de $15 \mathrm{~mL}$ (tubos A, B, C e D) foi transferida uma massa de 0,1000 $\mathrm{g}$ de MTK. No tubo A foram adicionados 5,00 $\mathrm{mL}$ de água deionizada (prova em branco), enquanto que aos tubos B, C e D foram adicionados $5,00 \mathrm{~mL}$ de solução contendo $\mathrm{Cr}$ (III) e Cr(VI), ambos em concentração de $0,050 \mathrm{mg} \mathrm{L}^{-1}$. Os tubos foram mantidos sob agitação em um agitador orbital por $10 \mathrm{~min}$ e centrifugados por $10 \mathrm{~min}$ a $2800 \mathrm{rpm}$. As fases líquidas foram filtradas e as fases sólidas reservadas para o estudo de dessorção. Uma lavagem do material MTK com água deionizada foi realizada, sendo que após agitação e centrifugação os sobrenadantes foram combinados e filtrados. As determinações de $\mathrm{Cr}$ (III) e $\mathrm{Cr}$ (VI) foram realizadas como já descrito, sendo determinada a concentração de $\mathrm{Cr}(\mathrm{VI})$ e de cromo total, obtendo-se a concentração de $\mathrm{Cr}$ (III) por diferença. Esse experimento foi repetido para a concentração de $0,500 \mathrm{mg} \mathrm{L}^{-1}$ de cada um dos íons. Para dessorção dos íons, a cada um dos tubos contendo MTK, foram adicionados 5,0 mL de $\mathrm{HNO}_{3}$ em concentração de $2,0 \mathrm{~mol} \mathrm{~L}^{-1}$, sendo os tubos mantidos sob agitação por 15 min e centrifugados por $10 \mathrm{~min}$. As amostras foram filtradas e nas fases líquidas foram determinadas as concentrações de $\mathrm{Cr}(\mathrm{VI})$ e de cromo total, sendo a concentração de $\mathrm{Cr}(\mathrm{III})$ obtida por diferença.

Os processos de sorção e de dessorção com os dois íons juntos foram repetidos, sendo utilizadas massas de MTK de 0,0250 e 0,0500 g.

\section{RESULTADOS E DISCUSSÃO}

\section{Tratamento e caracterização dos argilominerais}

Foi realizado o tratamento prévio da $\mathrm{MT}$ com $\mathrm{HNO}_{3}$ em concentração de 2,0 mol L-1, visando a remoção de impurezas, especialmente íons metálicos que poderiam interferir pela possível reação de outras espécies com a DFC. Essa elevada concentração de $\mathrm{HNO}_{3}$ foi inicialmente empregada, pois a etapa posterior de dessorção é crítica em decorrência da forte interação existente desse íon com fases minerais. ${ }^{18} \mathrm{O}$ tratamento com solução de $\mathrm{KCl}$ em concentração de $1,0 \mathrm{~mol} \mathrm{~L}^{-1}$ teve como objetivo a obtenção de um material constituído majoritariamente por íons $\mathrm{K}^{+}$(MTK) em sua camada interlamelar. A MTK mostrou uma distribuição granulométrica com $11 \%$ dos cristais menores que $63 \mu \mathrm{m} ; 11,7 \%$ entre 63 e $90 \mu \mathrm{m} ; 27,4 \%$ entre 90 e 125 $\mu \mathrm{m} ; 46 \%$ entre 125 e $180 \mu \mathrm{m}$ e $3,9 \%$ com partículas maiores que $250 \mu \mathrm{m}$. A distribuição granulométrica é uma informação relevante em estudos de sorção, uma vez que a interação do sorvente com o íon metálico não ocorre somente por troca iônica, mas também por interações superficiais. ${ }^{27}$ Dessa forma, menores tamanhos de grão expõem maiores áreas superficiais e, em consequência, um maior número de sítios de sorção.

O difratograma de raios-X da montmorilonita-K10 da Aldrich (MT) (Figura 1) apresentou um pico de baixa intensidade, identificado como MT em $2 \theta=8,86$ com (d001) correspondendo a um espaçamento basal de $9,98 \AA$, o que está concordante com a literatura, bem como a presença dos picos referentes à muscovita e ao quartzo. ${ }^{28,29}$ Ainda na Figura 1 é possível verificar que o difratograma da MT saturada com íons potássio praticamente não exibiu modificações, embora o pico de baixa intensidade correspondente à montmorilonita não tenha sido verificado, o que pode ser indicativo de algum efeito do tratamento ácido realizado. De acordo com a literatura, os tratamentos térmico e ácido realizados no processamento industrial desse material, destroem grande parte da estrutura cristalina característica da MT natural. ${ }^{28,29}$ Todavia, seria fundamental efetuar um estudo mais detalhado, visando investigar se houve destruição ou desordem na estrutura do material.

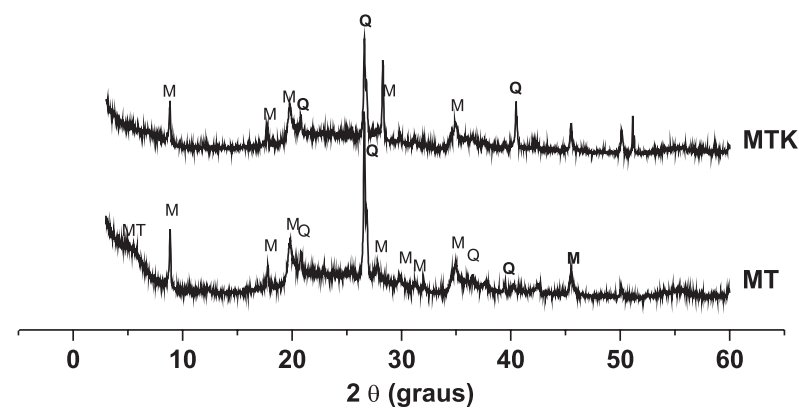

Figura 1. Padrões de difração de raios-X da amostra K-10(28152-2) natural (MT) e após tratamentos com soluções de $\mathrm{HNO}_{3} 2,0 \mathrm{~mol} \mathrm{~L}^{-1}$ e de $\mathrm{KCl} 1,0 \mathrm{~mol}$ $L^{-1}$ (MTK). A reflexão 001 da montmorilonita é indicada por MT e as reflexões do quartzo e muscovita são indicadas por $Q$ e $M$, respectivamente

\section{Método analítico}

Visando verificar a adequação do método analítico para posterior quantificação das concentrações de $\mathrm{Cr}(\mathrm{VI})$ e $\mathrm{Cr}(\mathrm{III})$, inicialmente foram avaliadas as condições ideais para elaboração das curvas analíticas para ambas as espécies de cromo. Deve-se enfatizar que o método oficial recomenda que o primeiro ponto da curva seja de $0,100 \mathrm{mg} \mathrm{L}^{-1} \cdot{ }^{26}$ Todavia, foram realizados experimentos empregando a concentração de $0,050 \mathrm{mg} \mathrm{L}^{-1}$ como primeiro ponto das curvas analíticas, tanto para $\mathrm{Cr}(\mathrm{III})$ como para $\mathrm{Cr}(\mathrm{VI})$. Essa concentração gerou 
um sinal de absorbância de $0,033 \pm 0,001$, considerado um nível de confiança de $95 \%$ para $n=6$, sugerindo que seria possível empregar esse ponto, em virtude do baixo desvio observado. Foram efetuadas triplicatas de curvas analíticas para $\mathrm{Cr}(\mathrm{VI})$ e para $\mathrm{Cr}(\mathrm{III})$, empregandose cinco soluções padrão com as seguintes concentrações: 0,050 ; 0,100; 0,200; 0,400 e 0,800 $\mathrm{mg} \mathrm{L}^{-1}$. Para $\mathrm{Cr}(\mathrm{VI})$, foram observados valores de $r=0,9999$, com equação da curva analítica $A=0,6272$ $C_{\mathrm{Cr}}+0,0020$ ao passo que para Cr(III) foram obtidos valores de $r=$ 0,9994 , e equação da curva analítica $A=0,6071 C_{\mathrm{Cr}}+0,0060(A=$ absorbância, $C_{\mathrm{Cr}}=$ concentração de cromo). Sob essas condições, os limites de detecção (LD) e de quantificação (LQ) observados foram, respectivamente, 0,008 e $0,03 \mathrm{mg} \mathrm{L}^{-1}$.

Uma vez que em amostras reais coexistem ambas as espécies no meio, surgiu o interesse de estudar a interferência de uma espécie iônica frente à outra. Dessa forma, foram realizados experimentos nas concentrações de 0,050 e $0,500 \mathrm{mg} \mathrm{L}^{-1}$ de $\mathrm{Cr}$ (III) e de $\mathrm{Cr}$ (VI) para a verificação de possíveis interferências na quantificação de uma das espécies, uma vez que um dos objetivos do estudo era a análise de especiação. Os resultados são mostrados na Tabela 1 .

Tabela 1. Determinação de $\mathrm{Cr}(\mathrm{VI})$ e $\mathrm{Cr}(\mathrm{III})$ em concentrações iniciais de 0,500 e $0,050 \mathrm{mg} \mathrm{L}^{-1}$. Nível de confiança de $95 \%, n=3$

\begin{tabular}{ccc}
\hline $\mathrm{Cr}(\mathrm{III})-\mathrm{mg} \mathrm{L}^{-1}$ & $\mathrm{Cr}(\mathrm{VI})-\mathrm{mg} \mathrm{L}^{-1}$ & Cromo total $-\mathrm{mg} \mathrm{L}^{-1}$ \\
\hline $0,050 \pm 0,007$ & $0,050 \pm 0,001$ & $0,100 \pm 0,008$ \\
$0,50 \pm 0,02$ & $0,50 \pm 0,03$ & $1,00 \pm 0,05$ \\
\hline
\end{tabular}

De acordo com a Tabela 1, os valores médios das triplicatas não expressam estimativas de desvios padrão significativos, para um nível de confiança de 95\%, para as amostras contendo $\mathrm{Cr}$ (III) e $\mathrm{Cr}(\mathrm{VI})$, mesmo em concentrações de $0,050 \mathrm{mg} \mathrm{L}^{-1}$. Sendo assim, a especiação do cromo nessas concentrações é possível e com sinais de desvios negligenciáveis. Cabe salientar que embora o método da DFC já seja utilizado para análises de água, ${ }^{26}$ a intenção de realizar esse estudo comparativo foi principalmente verificar a possibilidade de utilização em concentração inferior àquela recomendada $(0,100$ $\mathrm{mg} \mathrm{L}^{-1}$ ), bem como averiguar o nível de variabilidade do método para aplicação posterior aos estudos de sorção e de dessorção com a MTK. Esses resultados, aliados àqueles obtidos para as curvas analíticas, evidenciaram que seria possível o emprego do método analítico da DFC para estudar o processo de interação de íons cromo com a MTK, com ótima confiabilidade no que diz respeito à etapa de quantificação.

\section{Estudo de variáveis relacionadas ao processo de sorção e dessorção na MTK}

Um estudo da interação entre 0,1000 g de MTK e 10,00 mL de solução de $\mathrm{Cr}$ (III) foi realizado com tempos de 0,5, 10 e $60 \mathrm{~min}$. Constatou-se em todos os casos que a sorção de $\mathrm{Cr}$ (III) foi de cerca de $100 \%$, demonstrando uma ótima potencialidade da fase mineral na retenção desse íon, mesmo para o menor tempo de contato estudado. De acordo com a literatura, a interação entre Cr(III) com bentonita na forma sódica também mostrou tempo de contato relativamente curto para atingir um equilíbrio aparente, ao redor de 15 min, embora nesse caso o estudo tenha sido realizado com concentração inicial de $\mathrm{Cr}$ (III) de $130 \mathrm{mg} \mathrm{L}{ }^{-1} \cdot{ }^{30}$ Assim, é possível que o fato de haver uma maior disponibilidade de sítios no presente estudo pode ter sido a causa do processo de sorção ter sido muito rápido, sendo que a literatura relata situações de sorção de $\mathrm{Cr}$ (III) por bentonita praticamente instantâneas, ${ }^{31}$ assim como foi constatado no presente estudo.

Também foi verificado se um aumento da massa de MTK no processo de sorção e de dessorção de $\mathrm{Cr}$ (III) poderia comprometer os percentuais de sorção, bem como se poderia ocasionar sinais nas provas em branco. Estas mostraram sinais de absorbância entre 0,007 e 0,025 para massas de 0,1000 e 0,5000 g de MTK, respectivamente. Além disso, em estudos prévios de sorção de Cr(III) com essas massas foram obtidos resultados semelhantes, com remoção praticamente total para uma solução de $\mathrm{Cr}$ (III) em concentração inicial de 0,500 $\mathrm{mg} \mathrm{L}^{-1}$. A partir dessas observações foram realizados estudos com massas de 0,1000 g da MTK, o que proporcionou um processo de sorção em torno de $100 \%$ sem haver comprometimento dos sinais das provas em branco.

Um novo experimento de sorção foi realizado com concentrações de $\mathrm{Cr}(\mathrm{III})$ de 0,$250 ; 0,500$ e $1,000 \mathrm{mg} \mathrm{L}^{-1}$. Para as três situações, foram obtidos percentuais de remoção próximos de $100 \%$, como anteriormente. Em seguida, foram estudados os melhores tempos de contato sob agitação com solução de $\mathrm{HNO}_{3} 2,0 \mathrm{~mol} \mathrm{~L}{ }^{-1}$, para avaliar os processos de dessorção e de recuperação do $\mathrm{Cr}$ (III), para o estudo de sorção de $0,500 \mathrm{mg} \mathrm{L}^{-1}$ de $\mathrm{Cr}(\mathrm{III})$. Foram obtidos resultados variando entre 67 e $79 \%$ para 10 e 60 min de tempo de contato, respectivamente. Estes resultados evidenciam, uma vez mais, a forte interação existente entre a espécie $\mathrm{Cr}(\mathrm{III})$ e a fase mineral. Também foram realizados testes empregando o processo de dessorção em ultrassom durante 15 min, tendo sido obtidos percentuais de recuperação entre 66 e $77 \%$, demonstrando não haver melhora neste processo.

Embora as provas em branco não estejam diretamente relacionadas com o processo de dessorção de $\mathrm{Cr}$ (III) da MTK, essa etapa é bastante crítica, em virtude da elevada concentração de $\mathrm{HNO}_{3}$ empregada, que pode liberar íons metálicos, ou destruir parcialmente a fase mineral. Assim, foram realizados dois conjuntos de experimentos em diferentes dias, mantendo a MTK em contato com $\mathrm{HNO}_{3}$ 2,0 mol $\mathrm{L}^{-1}$ e empregando sete processos de dessorção sucessivos. A Figura 2 mostra os resultados para estes experimentos.

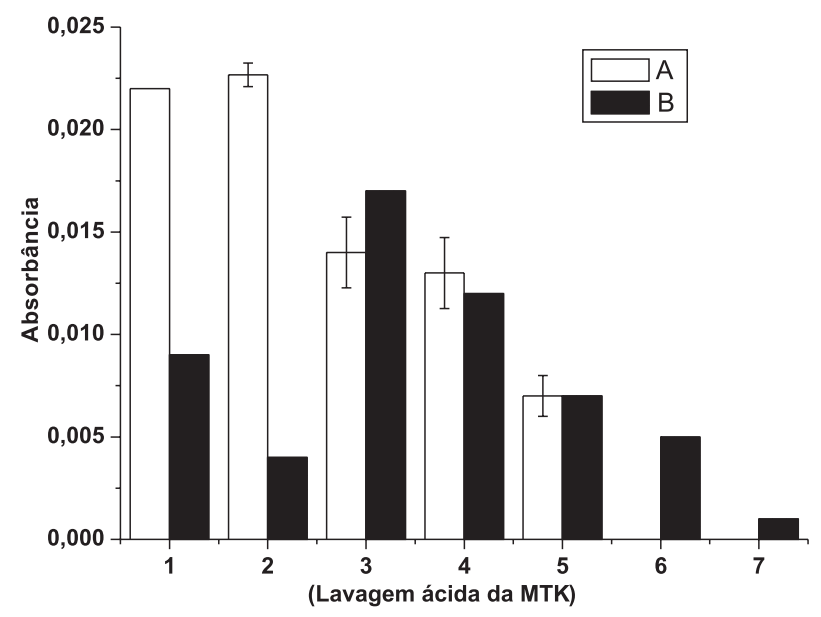

Figura 2. Valores de absorbância para as lavagens ácidas das amostras em branco, após tratamentos sucessivos por $15 \mathrm{~min}$ com 5,0 $\mathrm{mL}$ de $\mathrm{HNO}_{3} 2,0 \mathrm{~mol} \mathrm{~L}^{-1}$

Os sinais de absorbância obtidos para a MTK demonstraram uma ótima repetitividade para as amostras em branco realizadas em triplicata (A) e duplicata (B), conforme evidenciado pelas barras de desvios mostradas na Figura 2. Apesar de ocorrer um ligeiro aumento da absorbância na terceira dessorção, é possível notar que em seguida o sinal de absorbância exibido tendeu a uma diminuição significativa nos valores, sugerindo que o emprego desse tratamento torna o material mais puro, sem que haja liberação de material coloidal para a solução. A partir da sexta dessorção os sinais diminuem chegando próximo a zero na sétima dessorção, viabilizando o procedimento de dessorção com a MTK.

De acordo com a literatura, essa fase mineral é relativamente pura quando comparada com outras fases como vermiculita, por 
exemplo. ${ }^{21}$ Uma possível explicação para a redução gradativa do sinal obtido poderia estar relacionada ao teor de ferro dessa fase mineral, de $0,60 \%,{ }^{32}$ visto que íons Fe(III) são potenciais interferentes no método espectrofotométrico. ${ }^{26}$ Assim, as sucessivas lavagens poderiam estar liberando o ferro e por isso ocorreu uma gradativa diminuição no sinal analítico. Isso é reforçado por um estudo prévio (resultados não apresentados) empregando o argilomineral vermiculita (teor de Fe de 4,0\%), ${ }^{32}$ o qual propiciou sinais de absorbância muito elevados, provavelmente em decorrência da interferência de ferro, no método da DFC. Dessa forma, a MTK foi lavada sete vezes com $\mathrm{HNO}_{3} \mathrm{em}$ concentração de 2,0 $\mathrm{mol} \mathrm{L}^{-1}$ antes da continuidade do estudo.

\section{Estudo de sorção e de dessorção do $\operatorname{Cr}($ III) e $\operatorname{Cr}($ VI) em MTK}

A avaliação dos processos de sorção e dessorção de $\mathrm{Cr}$ (III) e Cr(VI), ambos em concentração inicial de $0,500 \mathrm{mg} \mathrm{L}^{-1}$, em tubos separados, bem como a prova em branco utilizando a MTK, foi realizada seguindo o procedimento descrito anteriormente. A prova em branco originária do processo de sorção ou de dessorção mostrou um sinal insignificante, justificando as etapas realizadas de tratamento ácido e sugerindo a ausência de potenciais interferentes na etapa de quantificação. $\mathrm{O} \mathrm{Cr}$ (III) foi retido quase que em sua totalidade na matriz de MTK, durante a sorção, apresentando um percentual de remoção de 98,8 \pm 0,7\% (nível de confiança de 95\%, $n=7$ ). Esse resultado é bastante satisfatório, evidenciando mais uma vez que a remoção de $\mathrm{Cr}$ (III) do meio aquoso é praticamente completa. Embora não tenha sido realizado ajuste de $\mathrm{pH}$ do meio, os valores monitorados durante o tempo de realização do experimento variaram de 4,7 a 5,5, tanto para esse estudo quanto para posteriores. Um aumento no $\mathrm{pH}$ também pode contribuir para um incremento na sorção do $\mathrm{Cr}$ (III). Isso pode estar relacionado com a presença de espécies como $\mathrm{Cr}(\mathrm{OH})_{2}{ }^{+}$ e $\mathrm{Cr}(\mathrm{OH})^{2+}$ que mesmo em meio ácido podem estar sendo sorvidas, além de haver uma menor competição entre os sítios de sorção pelo $\mathrm{H}^{+}$do meio, sendo demonstrado um considerável aumento na sorção em virtude da elevação do valor de $\mathrm{pH}$ de 4 para próximo de 5,2. ${ }^{30}$ $\mathrm{O}$ processo de precipitação também poderia estar contribuindo para auxiliar a retenção do $\mathrm{Cr}$ (III) com a elevação do $\mathrm{pH} .{ }^{21}$ Percentuais próximos de $100 \%$ de remoção de $\mathrm{Cr}$ (III) por bentonita também foram observados para valores de $\mathrm{pH}$ entre 5 e $7 .{ }^{31}$

A dessorção de $\mathrm{Cr}$ (III) da MTK foi $71 \pm 7 \%$ ( $n=7$, nível de confiança de $95 \%$ ). Diversos outros experimentos foram realizados no sentido de tentar aprimorar esse processo de dessorção, tendo sido variados os tipos de ácidos, as concentrações, o uso de ultrassom e a elevação da temperatura até $60^{\circ} \mathrm{C}$; porém, em todo os casos, os resultados se mostraram aquém do esperado. Por outro lado, ao ser avaliado o processo de interação entre $\mathrm{Cr}(\mathrm{VI})$ e a MTK, $93 \pm 3 \%$ (n $=3$, nível de confiança de $95 \%$ ) do Cr(VI) permaneceu em solução. Isso ocorre em virtude do argilomineral estar carregado negativamente, especialmente em valores de $\mathrm{pH}$ próximos da neutralidade ou tendendo à faixa básica, o que propicia uma repulsão eletrostática para o $\mathrm{Cr}(\mathrm{VI})$, ao contrário do que ocorre com o $\mathrm{Cr}$ (III). ${ }^{18}$

Em seguida aos experimentos realizados com os íons cromo separadamente, o próximo passo foi estudar o comportamento das duas espécies juntas. Em uma primeira etapa o $\mathrm{Cr}(\mathrm{VI})$ da fase sobrenadante foi quantificado utilizando-se o método da DFC e, posteriormente, o $\mathrm{Cr}$ (III) sorvido foi dessorvido em meio $\mathrm{HNO}_{3} 2,0 \mathrm{~mol} \mathrm{~L}^{-1}$ e quantificado pelo mesmo método após etapa de oxidação. Na Tabela 2 são mostrados os resultados obtidos para o estudo realizado sendo variada a massa de MTK para concentrações iniciais de $\mathrm{Cr}(\mathrm{III})$ e de $\mathrm{Cr}(\mathrm{VI})$ de $0,500 \mathrm{mg} \mathrm{L}^{-1}$. Cabe salientar que em virtude dos ótimos percentuais de sorção de $\mathrm{Cr}$ (III) para a massa inicial de $0,1000 \mathrm{~g}$, foi estudado o uso de massas inferiores de MTK, visando verificar se o mesmo desempenho seria mantido.
Tabela 2. Valores dos percentuais de sorção e de dessorção de $\operatorname{Cr}(\mathrm{III})$ e percentual de $\mathrm{Cr}$ (VI) mantido em solução. Concentração inicial de $\mathrm{Cr}(\mathrm{III})$ e de $\operatorname{Cr}(\mathrm{VI})=0,500 \mathrm{mg} \mathrm{L}^{-1}$, na presença de diferentes massas de MTK. Nível de confiança de $95 \%, n=3$

\begin{tabular}{lcrc}
\hline & $0,1000 \mathrm{~g}$ & $0,0500 \mathrm{~g}$ & $0,0250 \mathrm{~g}$ \\
\hline Percentual de sorção de Cr(III) & $99 \pm 2$ & $101 \pm 1$ & $100 \pm 3$ \\
Percentual de dessorção de Cr(III) & $82 \pm 2$ & $85 \pm 9$ & $89 \pm 13$ \\
Percentual de Cr(VI) em solução & $96 \pm 3$ & $94 \pm 7$ & $98 \pm 2$ \\
\hline
\end{tabular}

Conforme mostra a Tabela 2 , foram verificados percentuais de sorção entre 99 e $101 \%$ para as três diferentes massas estudadas. De acordo com os desvios observados, pode-se constatar que não há diferenças significativas em decorrência da diminuição da massa de MTK. Embora não tenha sido determinada a capacidade máxima de sorção de $\mathrm{Cr}$ (III) pela MTK no presente estudo, com base no valor de capacidade de troca catiônica desse material, de $0,59 \mathrm{mmol} \mathrm{g}^{-1},{ }^{32}$ pode-se inferir que mesmo para a menor massa de MTK de $25 \mathrm{mg}$, haveria a disponibilidade para sorver cerca de $0,77 \mathrm{mg}$ de $\mathrm{Cr}$ (III) sendo que a massa de $\mathrm{Cr}$ (III) contida no volume de $10,00 \mathrm{~mL}$ é de $5 \mu \mathrm{g}$; muito abaixo, portanto, do valor máximo disponível, o que aliado ao forte processo de interação existente explica os elevados percentuais de sorção observados. Baseado no valor de CTC de $0,59 \mathrm{mmol} \mathrm{g}^{-1}$ haveria a possibilidade de sorver cerca de 31 $\mathrm{mg}$ de $\mathrm{Cr}(\mathrm{III})$ por grama de MTK, sendo que a literatura cita valores da ordem de 61 a $118 \mathrm{mg} \mathrm{g}^{-1},{ }^{30} \mathrm{e} \mathrm{de} \mathrm{44,4} \mathrm{a} \mathrm{49,8} \mathrm{mg} \mathrm{g}^{-1},{ }^{33}$ nos dois casos para bentonitas. Na Tabela 2 são mostrados também os resultados observados para o processo de dessorção em $\mathrm{HNO}_{3} 2,0 \mathrm{~mol} \mathrm{~L}^{-1}$ do $\mathrm{Cr}$ (III) que foi previamente sorvido, tendo sido obtidos percentuais de 82,85 e $89 \%$. Os resultados sugerem haver uma melhora no percentual de dessorção de $\mathrm{Cr}$ (III) com a diminuição da massa de MTK, embora os valores dos desvios tenham sido relativamente elevados. Isso poderia estar relacionado com a menor quantidade de sítios disponíveis para a menor massa de MTK, facilitando a remoção por parte do $\mathrm{HNO}_{3}$. Essa dificuldade de remoção do $\mathrm{Cr}$ (III) deve estar relacionada com a forte interação existente entre esse íon e a MTK, ${ }^{18}$ o que de certa forma dificulta utilizar a MTK como um material para determinação de $\mathrm{Cr}(\mathrm{III})$, ou para realizar a etapa de pré-concentração dessa espécie. Assim como havia sido constatado anteriormente, praticamente não houve sorção de $\mathrm{Cr}(\mathrm{VI})$ pela MTK. Conforme apresentado na Tabela 2, não parece haver influência da massa de MTK na sorção de Cr(VI), sendo que foram observados valores de 94 a $98 \%$ de $\mathrm{Cr}(\mathrm{VI})$ que foi mantido em solução. Estudos envolvendo a sorção de $\mathrm{Cr}(\mathrm{VI})$ em bentonita demonstram claramente o aumento da sorção dessa espécie de cromo com a diminuição do valor de $\mathrm{pH}$, tendo sido observados percentuais de sorção de $\mathrm{Cr}(\mathrm{VI})$ próximos de $20 \%$ para valores de $\mathrm{pH}$ entre 4,5 e 5,5, bem como não haver sorção significativa de $\mathrm{Cr}(\mathrm{VI})$ acima de $\mathrm{pH} 6 .^{31}$ No presente estudo, para a mesma faixa de $\mathrm{pH}$, os valores máximos observados para a sorção de $\mathrm{Cr}(\mathrm{VI})$ ficaram entre 2 e $6 \%$, de acordo com a Tabela 2. Conforme Fritzen et al., ${ }^{34}$ a interação entre montmorilonita e $\mathrm{Cr}(\mathrm{VI})$ é favorecida com a diminuição no valor de $\mathrm{pH}$ entre 1 e 2, sendo demonstrada uma sorção mais significativa em $\mathrm{pH}$ 1. De acordo com os autores, foi necessário um tempo de contato de $12 \mathrm{~h}$, devido ao processo ser lento, provavelmente em decorrência da estrutura altamente porosa da montmorilonita. Aliado ao valor de $\mathrm{pH}$, essa questão cinética também foi favorável para o presente estudo, cujo tempo de contato foi de $10 \mathrm{~min}$, minimizando, portanto, a sorção da espécie hexavalente.

Em virtude dos resultados apresentados, foi verificado se em menores concentrações de $\mathrm{Cr}$ (III) e $\mathrm{Cr}(\mathrm{VI})$ poderia haver o mesmo nível de confiabilidade analítica. Para tal, foi testada a concentração de $0,050 \mathrm{mg} \mathrm{L}^{-1}$ na presença das massas de 0,1000 e $0,0250 \mathrm{~g}$ de MTK, sendo os resultados apresentados na Tabela 3. 
Tabela 3. Valores dos percentuais de sorção e de dessorção de $\mathrm{Cr}$ (III) e percentual de $\mathrm{Cr}(\mathrm{VI})$ mantido em solução. Concentração inicial de $\mathrm{Cr}(\mathrm{III})$ e de $\mathrm{Cr}(\mathrm{VI})=0,050 \mathrm{mg} \mathrm{L}^{-1}$, na presença de diferentes massas de MTK. Nível de confiança de $95 \%, n=3$

\begin{tabular}{lcc}
\hline & $0,1000 \mathrm{~g}$ & $0,0250 \mathrm{~g}$ \\
\hline Percentual de sorção de Cr(III) & $99 \pm 2$ & $104 \pm 9$ \\
Percentual de dessorção de Cr(III) & $81 \pm 3$ & $86 \pm 9$ \\
Percentual de Cr(VI) em solução & $96 \pm 3$ & $98 \pm 8$ \\
\hline
\end{tabular}

Novamente pode ser verificado que não houve influência da massa de MTK, assim como não houve diferenças significativas em relação aos resultados apresentados na Tabela 2 para o processo de sorção de $\mathrm{Cr}(\mathrm{III})$. De modo análogo, os resultados de dessorção em $\mathrm{HNO}_{3}$ e de $\mathrm{Cr}(\mathrm{VI})$ mantido em solução foram bastante similares àqueles apresentados para a concentração inicial de $0,500 \mathrm{mg} \mathrm{L}^{-1}$ (Tabela 2), demonstrando que o processo parece ser independente da massa utilizada, bem como das diferentes concentrações de $\mathrm{Cr}$ (III) ou de $\mathrm{Cr}(\mathrm{VI})$.

\section{CONCLUSÕES}

Os resultados apresentados por difração de raio $\mathrm{X}$, aliados àqueles mostrados na literatura para a montmorilonita $\mathrm{K}-10$ oriunda da Aldrich, sugerem que esse material não reúne características de montmorilonita, provavelmente em decorrência do tratamento térmico e do meio ácido empregado, embora uma avaliação mais minuciosa deva ser conduzida, para uma melhor elucidação. Apesar disso, esse material exibiu uma excelente interação com $\mathrm{Cr}(\mathrm{III}), \mathrm{o}$ qual foi retido aproximadamente em $100 \%$ pela MTK nas diferentes situações estudadas. A interação existente parece ser muito intensa, sendo que mesmo ácido nítrico em elevada concentração não propiciou uma recuperação completa (máximo de $89 \%$ ), para as menores massas de MTK. Isso sugere que utilizar a MTK como uma forma de pré-concentração analítica de $\mathrm{Cr}$ (III) seria apropriado no que diz respeito ao processo de sorção, porém haveria uma limitação, pois a recuperação e, consequentemente, os estudos de pré-concentração não seriam satisfatórios. Uma possibilidade de melhorar o processo de dessorção do $\mathrm{Cr}$ (III) seria o emprego de agentes complexantes como citrato conforme citado na literatura, ${ }^{35}$ o que, contudo, não foi avaliado no presente estudo. Por outro lado, a forma $\mathrm{Cr}(\mathrm{VI})$ não demonstrou interação com a MTK, sob as condições de $\mathrm{pH}$ estudadas, para as concentrações iniciais de 0,050 e $0,500 \mathrm{mg} \mathrm{L}^{-1}$.

Dessa forma, em decorrência dos elevados percentuais de sorção de $\mathrm{Cr}$ (III) do meio aquoso e a permanência de praticamente todo o $\mathrm{Cr}(\mathrm{VI})$ em solução, pode-se inferir que é viável o emprego da MTK como uma fase para especiação entre as duas espécies estudadas. Isso pode ser particularmente útil para a utilização com técnicas analíticas que requerem a separação prévia das espécies como a espectrometria de absorção ou de emissão atômica.

\section{AGRADECIMENTOS}

Ao CNPq pelo suporte financeiro.

\section{REFERÊNCIAS}

1. Kabata-Pendias, A.; Trace elements in soils and plants, $3^{\text {rd }}$ ed., CRC Press: Boca Raton, 2001, p. 413.

2. Zhang, N.; Suleiman, J. S.; He, M.; Hu, B.; Talanta 2008, 75, 536

3. Sperling, M.; Yin, X.; Welz, B.; Analyst 1992, 117, 629.

4. Themelis, D. G.; Kika, F. S.; Economou, A.; Talanta 2006, 69, 615.

5. Matos, W. O.; Nóbrega, J. A.; Souza, G. B.; Nogueira, A. R. A.; Quim. Nova 2008, 31, 1450 .

6. Anderson, R. A. J.; Am. Coll. Nutr. 1998, 17, 548.

7. Elci, L.; Kartal, A. A.; Soylak, M.; J. Hazard. Mater. 2008, 153, 454.

8. Aydin, F. A.; Soylak, M.; J. Hazard. Mater. 2009, 162, 1228.

9. Zayed, A. M.; Terry, N.; Plant Soil 2003, 249, 139.

10. CONAMA (Conselho Nacional do Meio Ambiente); Resolução $n^{\circ} 397$, de 03 de abril de 2008.

11. Sussulini, A.; Arruda, M. A. Z.; Eclética Quím. 2006, 31, 73.

12. Uluozlu, O. D.; Tuzen, M.; Soylak, M.; Food Chem. Toxicol. 2009, 47, 2601.

13. Rajesh, N.; Jalan, R. K.; Hotwany, P. J.; J. Hazard. Mater. 2008, 150, 723.

14. Chang, X.; Li, Z.; Cui, Y. Zhu, X.; Zang, Z.; Microchem. J. 2008, 90, 71.

15. Pereira, C. D.; Techy, J. G.; Quináia, S. P.; Ganzarolli, E. M.; Can. J. Anal. Sci. Spectrosc. 2009, 53, 275.

16. Sposito, G.; The Chemistry of Soils, Oxford University Press: New York, 1989.

17. Tan, K. H.; Principles of soil chemistry, $2^{\text {nd }}$ ed., Dekker: New York, 1993.

18. McBride, M. B.; Environmental Chemistry of soils, Oxford University Press: New York, 1994.

19. de Aguiar, M. R. M. P.; Novaes, A. C.; Guarino, A. W. S.; Quim. Nova 2002, 25, 1145.

20. Abollino, O.; Giacomino, A.; Malandrino, M.; Mentasti, E.; Appl. Clay Sci. 2008, 38, 227.

21. Bhattacharyya, K. G.; Gupta, S. S.; Adv. Colloid Interface Sci. 2008, $140,114$.

22. Akta, Y. K.; Fresenius Environ. Bull. 2005, 14, 993.

23. Akta , Y. K.; Hilmi bar, H.; Fresenius Environ. Bull. 2005, 14, 983.

24. Rajesh, N.; Mishra, B. G.; Pareek, P. K.; Spectrochim. Acta, Part A 2008, 69,612 .

25. Afzali, D.; Mostafavi, J.; J. AOAC Int. 2008, 91, 1430.

26. APHA - American Public Health Association, Water Environment Federation, American Water Works Association, Water Pollution Control Federation, Washington, DC, 1995.

27. Gomes, C. F.; Argilas - O que são e para que servem, Fundação Calouste Gulbenkian: Lisboa, 1986.

28. Villegas, R. A. S.; Santo Júnior, J. L. E.; Mattos, M. C. S.; Aguiar, M. R. M. P.; Guarino, A. W. S.; J. Braz. Chem. Soc. 2005, 16, 565.

29. Pinto, A. C.; Oliveira, C. H.; Ribeiro, N. M.; Quim. Nova 2008, 31, 562.

30. Ghorbel-Abid, I.; Jrad, A.; Nahdi, K.; M. Trabelsi-Ayadi, M.; Desalination 2009, 246, 595.

31. Khan, S. A.; Rehman, R.; Khan, M. A.; Waste Manage. 1995, 15, 271.

32. Abate, G.; Masini, J. C.; J. Agric. Food Chem. 2005, 53, 1612.

33. Alvarez-Ayuso, E.; Garcia-Sanchez, A.; Clays Clay Miner. 2003, 51, 475.

34. Fritzen, M. B.; Souza, A. J.; Silva, T. A. G.; Souza, L.; Nome, R. A.; Fiedler, H. D.; Nome, F.; J. Colloid Interface Sci. 2006, 296, 465.

35. Westrup, J. L.; Fritzen, M. B.; Souza, A. J.; Bedendo, G. C.; Nome, F.; Fiedler, H. D.; J. Braz. Chem. Soc. 2005, 16, 982. 